\title{
Comparative study of the pathogenicity and humoral response of Lomentospora, Scedosporium and Aspergillus infections in a murine model
}

Idoia Buldain, Leire Martin-Souto, Aize Pellon, Aitziber Antoran, Aitor Rementeria, Fernando L. Hernando and Andoni Ramirez-Garcia Fungal and Bacterial Biomics Research Group., Dept. of Immunology, Microbiology \& Parasitology, University of the Basque Country (UPV/EHU), Leioa, Spain

\section{INTRODUCTION}

Lomentospora (Scedosporium) prolificans is an emerging pathogen that causes, above all, disseminated infections in immunocompromised patients with very high mortality rates. This is mainly associated with the kind of infections, the fungal intrinsic drug resistance and the difficulties to perform an accurate and rapid diagnosis. Therefore, an improvement in diagnostic tools, resulting from the identification of new markers or antigens, is essential to establish a proper treatment and reduce the unacceptable morbimortality caused by this fungus.

\section{OBJECTIVE}

To compare the pathogenicity of Lomentospora prolificans, Scedosporium apiospermum, S. aurantiacum and Aspergillus fumigatus in a murine model, and to study the cross-reactivity with $L$. prolificans of the serum IgGs from mice infected with the other fungal species, identifying the major antigens.

\section{RESULTS}

\section{Murine infection by Lomentospora, Scedosporium and Aspergillus fungi}

\section{Methods}

Swiss female 8-week-old immunocompetent mice were infected intravenously with $10^{5}$ conidia/animal of L. prolificans, S. apiospermum, $S$. aurantiacum and $A$. fumigatus (clinical isolates). Twenty eight days after infection, animals were sacrificed for blood and organ extraction.
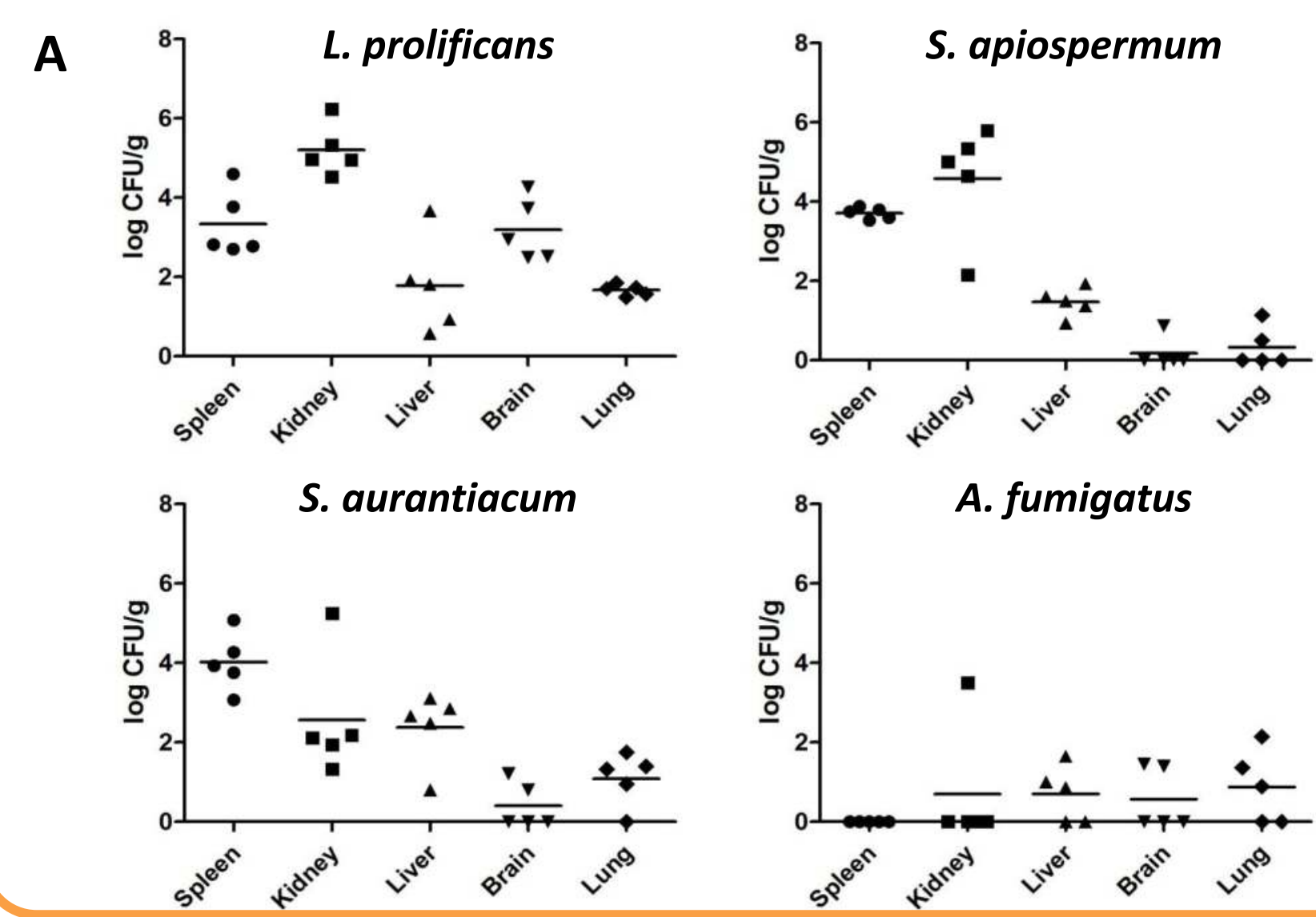

\section{Results}

Mice infected with Lomentospora and Scedosporium species developed signs associated with infection, while no signs were observed in $A$. fumigatus group. Survival rates were $50 \%$ in L. prolificans group, $\mathbf{8 3 . 3 \%}$ in $S$. apiospermum and S. aurantiacum, and $100 \%$ in $A$. fumigatus. In Lomentospora and Scedosporium infected groups high CFU counting were obtained, while in $A$. fumigatus group very few CFUs were collected (Fig. $1 \mathrm{~A})$. Moreover, the histological analysis showed renal affectation on Lomentospora/Scedosporium groups (Fig. 1B).
B L. prolificans

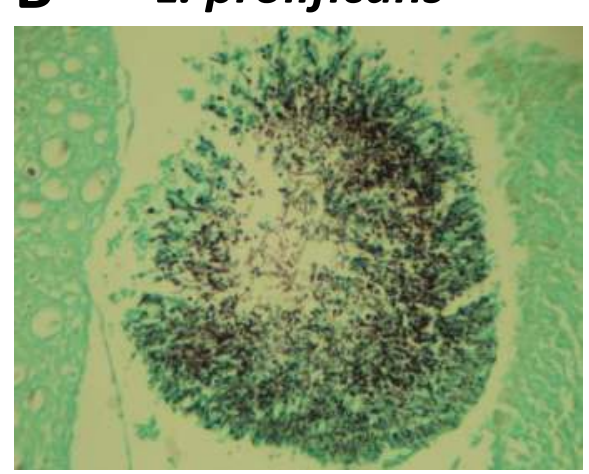

S. apiospermum

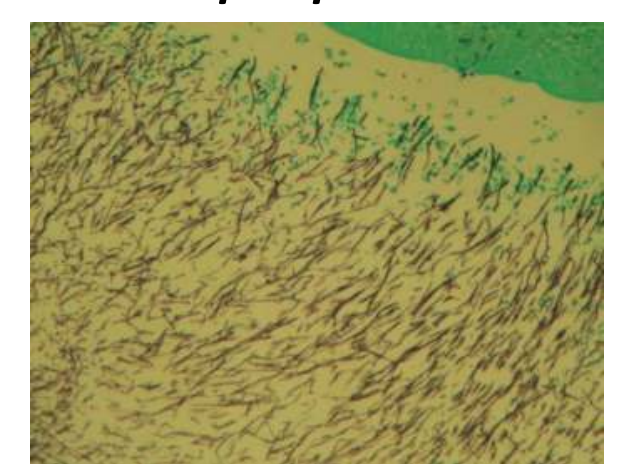

S. aurantiacum

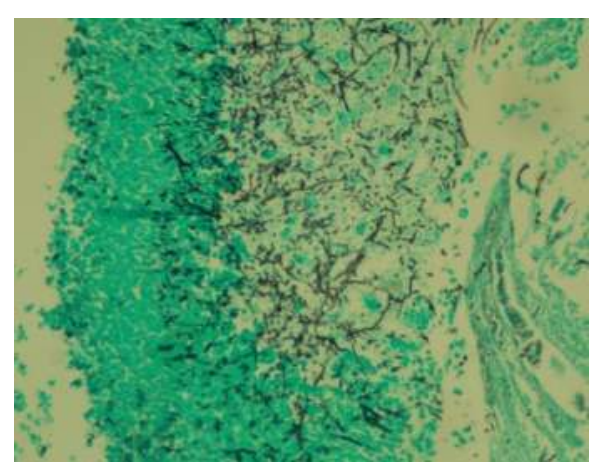

Fig. 1. Number of CFU/g of mice infected with Lomentospora, Scedosporium and Aspergillus (A); and histological study of kidneys of mice infected with Lomentospora/Scedosporium (B).

\section{Cross-reativity study of the humoral response}

\section{Methods}

Pooled sera of 5 mice of each group were used over L. prolificans cell extract proteome (hyphae and conidia) to detect antigens by immunoblotting (Fig. 2). The most important antigens of L. prolificans were identified by LC-MS/MS (Table 1 ).

Table 1. Identified antigens of $L$. prolificans in total cell extract.

\begin{tabular}{cc}
\hline Spot & Identified protein \\
\hline $\mathbf{1 , 2}$ & Heat shock protein 70 \\
3 & Hypothetical protein \\
& SAPIO_CDS2096 \\
$4,5,6$ & Proliferating cell nuclear \\
& antigen
\end{tabular}

$7,8,9,10 \quad$ Heat shock protein 70 (fractions)

A L. prolificans

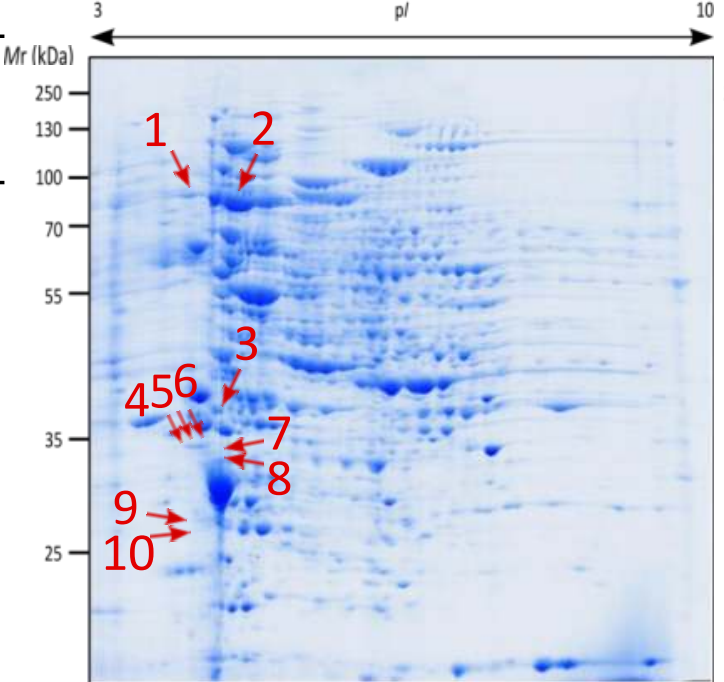

\section{Results}

The Heat shock protein 70 (Hsp70) was identified as the major antigen, being identified in different spots as the full protein or fragments of it.

All the most immunoreactive antigens showed cross-reactivity with the sera from mice infected with Scedosporium species but not with Aspergillus, which showed a completely different immunome pattern (Fig. 2).

B L. prolificans

C Scedosporium spp.

D

A. fumigatus
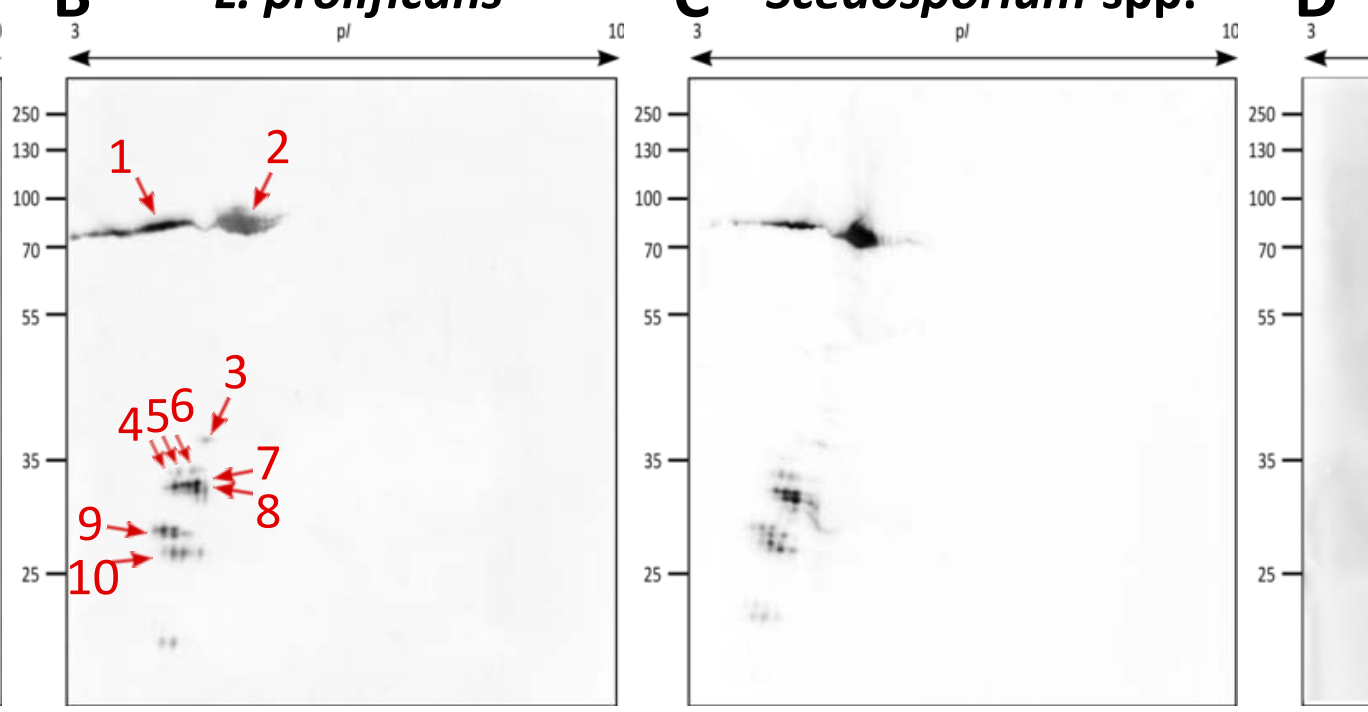

Fig. 2 Proteome of $L$. prolificans protein total extract $(A)$, and immunome using the pooled sera of mice infected with L. prolificans (B), Scedosporium spp. (C) and A. fumigatus (D). Marked spots were identified by LC-MS/MS.

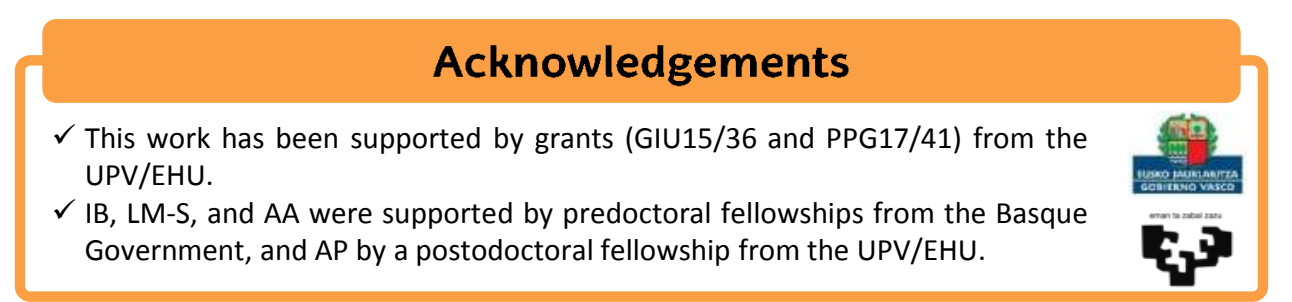

\section{CONCLUSIONS}

- Hsp70 stands out as interesting candidate to be evaluated as diagnostic marker of infections caused by Lomentospora/Scedosporium. 